\title{
Filtering of behaviourally relevant temporal parameters of a grasshopper's song by an auditory interneuron
}

\author{
Bernhard Ronacher and Andreas Stumpner* \\ Institut für Zoologie II, Universität Erlangen-Nürnberg, Staudtstrasse 5, D-8520 Erlangen, Federal Republic of Germany
}

Accepted February 29, 1988

Summary. In females of the acridid grasshopper Chorthippus biguttulus, thoracic auditory interneurons were investigated with respect to their selectivity for temporal parameters of the conspecific song. Special attention was given to the detection of small gaps in the "syllables' of the song, since behavioural experiments have shown that the presence or absence of gaps is critical for the female's Innate Releasing Mechanism (cf. Fig. 1).

The spiking response of one ascending interneuron, the AN4, shows filtering properties which closely resemble the behavioural reactions (cf. Figs. 1, 3 and $5 \mathrm{~b}$ ). The difference in the AN4's reaction to stimuli with gaps and uninterrupted stimuli is maintained over the behaviourally relevant intensity range (Fig. 4). This reaction is reliable enough that the stimulus type could be inferred by higher centres even from single stimulus presentations. Hence, this neuron is likely to participate in the task of gap detection and probably is a part of the neuronal filter network which determines the characteristics of the Innate Releasing Mechanism of this species. However, this interneuron is not species-specific: A homologue exists in other acridids as well and, in Locusta migratoria, has similar response characteristics (Fig. 6). The inferences of this observation for the evolution of an Innate Releasing Mechanism are discussed.

\section{Introduction}

In many acridid grasshoppers, the sexual partners are attracted to each other by means of an elabo-

Abbreviations: CNS central nervous system; PST-histogram post-stimulus-time-histogram; SPL sound pressure level; IRM Innate Releasing Mechanism

* To whom offprint requests should be sent rate acoustic communication. The production and recognition of the conspecific song constitute an important barrier against hybridisation (Perdeck 1957). The filtering properties of the recognition process (Innate Releasing Mechanism, IRM) have been thoroughly investigated by behavioural experiments with artificial stimuli in the acridid grasshopper species Chorthippus biguttulus (D. von Helversen 1972; D. and O. von Helversen 1975, 1983; O. von Helversen 1979). However, our knowledge of how such filters are realized in the CNS is rather limited. Only in few species neurons have been described with low pass, high pass and band pass characteristics for temporal parameters of the conspecific song (crickets: Schildberger 1984; frogs: Rose and Capranica 1983, 1984; Walkowiak 1984).

We investigated auditory interneurons in the CNS of Ch. biguttulus females with respect to their processing of behaviourally relevant temporal song parameters. In this paper, we focus upon one outstanding characteristic of the IRM of this species: Ch. biguttulus females respond very well to a model song consisting of 20 'syllables' of white noise (each $80 \mathrm{~ms}$ in duration) with interspersed pauses of $20 \mathrm{~ms}$ duration (see Fig. 1, upper inset). However, if gaps greater than $2-3 \mathrm{~ms}$ in duration are inserted into the syllables, the effectiveness of this modified model is destroyed completely (see D. von Helversen 1972; O. von Helversen 1979; cf. also Fig. 1).

The neuronal filter of the IRM, therefore, must be able to detect such small gaps in a noisy acoustic signal. This task appears to present problems even at the most peripheral level, the tympanic receptor fibres. An investigation of auditory receptors in Ch. biguttulus revealed that a single receptor fibre is not able to transmit the information about gaps in the relevant time range (Ronacher and Römer 
1985). Instead it has been proposed that the presence of gaps can be inferred by the CNS from a gap-induced synchronization of spikes on parallel receptor fibres (Ronacher and Römer 1985; cf. also Michelsen et al. 1985; Surlykke et al. 1988).

On the basis of this information, a subsystem of the CNS must decide whether a stimulus (model song) is sufficiently similar to the conspecific song and, therefore, should be accepted or not. There is evidence that the final decision takes place within the brain (supraoesophageal ganglion) (Bauer and O. von Helversen 1987; cf. also Elsner and Huber 1969; Ronacher et al. 1986). To our present knowledge, receptor fibres usually do not ascend further than the prothoracic ganglion (Rehbein et al. 1974; Römer 1985). Thus, we asked how the information about the presence or absence of small gaps is transmitted from the thoracic ganglia to the supraoesophageal ganglion. Here we describe the reactions of an identified ascending interneuron which is a likely candidate for the filtering and transmission of information in this special context.

\section{Material and methods}

The animals used in the experiments were females of Chorthippus biguttulus L. caught in the field in southern Germany, and both sexes of Locusta migratoria L., obtained from a commercial supplier. Intracellular recordings were made with glass microelectrodes whose tips were filled with a $3-5 \%$ solution of Lucifer Yellow (in $0.5 \mathrm{M} \mathrm{LiCl}$ ). After physiological characterization the cells were filled with Lucifer. Stained neurons were drawn from wholemounts after fixation in $4 \%$ paraformaldehyde, dehydration and clearing in methyl salicylate (cf. Stewart 1978). The experiments were performed in an anechoic Faraday cage. The animals were anesthetized for dissection by brief exposure to $\mathrm{CO}_{2}$ and then attached either with wax colophonium or with minute insect pins to a free-standing holder (thickness $4 \mathrm{~mm}$ ). Head, gut, wings and legs were removed. The thorax was opened dorsally, the metathoracic ganglion was exposed and, in some of the preparations, desheathed. To ensure stability, the metathoracic ganglion was held on a nickel-chrome spoon and covered with locust Ringer solution (Pearson and Robertson 1981). The electrode was inserted into the metathoracic ganglion or into the connectives between meso- and metathoracic ganglion.

The stimulation program was controlled by a microcomputer (AIM 65, Rockwell). The computer generated the amplitude modulation of the stimuli; the digital signal was converted by a DAC-element. The resulting envelope was multiplied by white noise (3-40 kHz). The amplified signal reached the loudspeaker via an attenuator. Stimuli could be presented either on the left or on the right side of the preparation by means of a computer-controlled switch. The distance between loudspeakers and preparation was $30 \mathrm{~cm}$. Sound pressure was measured with a Brüel \& Kjaer amplifier 2606 and a 1/2" Brüel \& Kjaer microphone situated at the preparation site, and is given in $\mathrm{dB}$ re $2 \times 10^{-5} \mathrm{~N} / \mathrm{m}^{2}$ (dB SPL). Usually the stimuli consisted of two 'syllables' separated by a pause of $25 \mathrm{~ms}$ and were presented to the animal at a rate of $1.1 / \mathrm{s}$. Each syllable was composed of 11 pulses and 10 gaps. Behavioural experi-

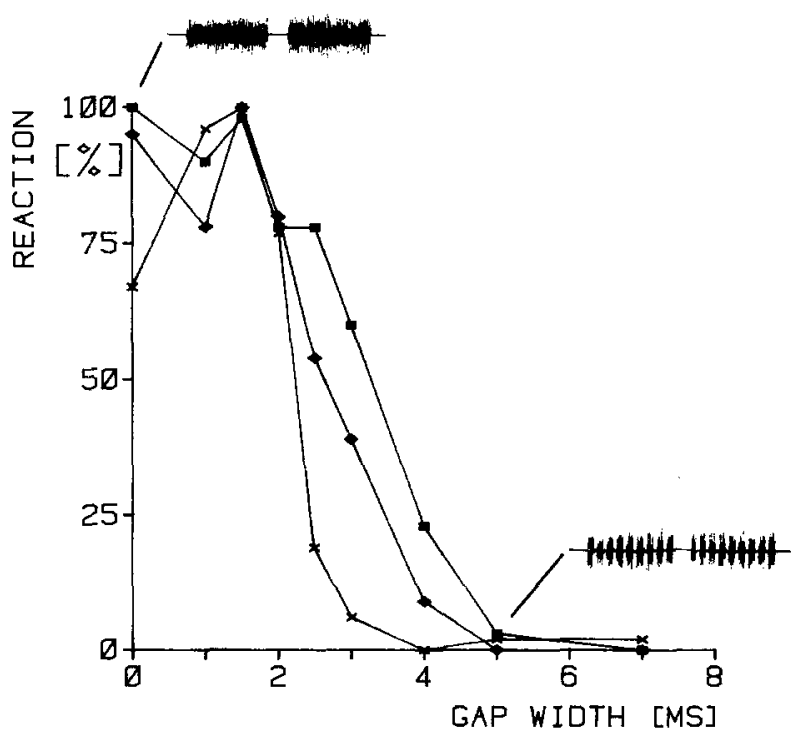

Fig. 1. Behavioural reaction of $3 \mathrm{Ch}$. biguttulus females to model songs of rectangularly modulated white noise. A detail of the temporal structure of the models is indicated by the insets, representing syllables without gaps and syllables with $5 \mathrm{~ms}$ gaps. Model songs for behavioural test consisted of 50 syllables. Temperature during tests: $23-25^{\circ} \mathrm{C}$. Sound pressure $70 \mathrm{~dB}$ SPL. $n=54(\mathbf{m} \bullet)$ or $159(\times)$ per data point. Data kindly provided by $D$. and $O$. von Helversen

ments have shown that the 'detectable' gap width also depends on the duration of the pulses between the gaps (D. von Helversen 1972). Therefore, in order not to confuse two effects, in an experimental series only the gap width was varied, while the duration and number of pulses within a syllable were constant. In these experiments the syllable duration varied between 90 and $150 \mathrm{~ms}$ (with $7.5 \mathrm{~ms}$ pulse duration). To allow a comparison of spike responses to different stimuli, the spikes were counted only in a time window which was adjusted to the shortest syllable type of a series (in the above mentioned example: $90 \mathrm{~ms}$ (+10 ms latency) after stimulus onset).

Electrophysiological experiments were performed at room temperature $\left(22-25^{\circ} \mathrm{C}\right)$. Temperature was monitored in the experimental chamber, near the animal. Behavioural tests were performed in a computer controlled set up; for details see $O$. von Helversen (1979).

\section{Results}

\section{Behavioural results}

The behavioural reactions of 3 Ch. biguttulus females to model songs containing gaps of different widths are shown in Fig. 1. Depending on the individual, the behavioural reaction drops below $25 \%$ of the maximum value at gap widths between 2.5 and $4 \mathrm{~ms}$. It should be noted that these experiments were performed at room temperature $\left(23-25^{\circ} \mathrm{C}\right)$ in order to allow a direct comparison with neurophysiological results. At higher temperatures $\left(30-35^{\circ} \mathrm{C}\right)$ the steep decrease of the female's reac- 
tion occurs even with gaps of 1-2 ms duration (O. von Helversen 1979). The critical gap width also depends upon the sound intensity and upon the length of pulses between gaps (cf. D. von Helversen 1972; O. von Helversen 1979).

\section{Interneuron morphology}

The morphology of an ascending interneuron is shown in Fig. 2 a. The soma is located ventrally near the midline of the ganglion. A primary neurite runs in a lateral loop dorsally and crosses the midline of the ganglion in medial height. An ascending axon and a main dendrite originate from this point and run contralaterally in the frontal direction (the terms ipsilateral and contralateral are used with respect to the soma). The dense branches which arise from the main dendrite expand mainly on the contralateral side of the frontal auditory neuropil.

This neuron's structure is obviously homologous to the AN4-neuron described in Locusta migratoria (compare Fig. $2 \mathrm{a}$ and b; see also Römer and Marquart 1984; synonymous: neuron 541, Boyan 1986). We will use the term AN4 for Ch. biguttulus, too. A similar neuron with a small ipsilateral dendritic branch has also been described in Omocestus viridulus L. (TH3-AC3, Hedwig 1986). This TH3-AC3 is probably also an homologue of the AN4 since we found such an ipsilateral dendrite in some individuals of Ch. biguttulus and L. migratoria, too.

\section{Interneuron response}

This neuron is more sensitive to contralateral stimulation, although the directionality is not pronounced. The spike responses of the AN4-neuron of a Ch. biguttulus female to syllables with gaps of different width are shown in Fig. 3. The neuron reacted with circa 12.5 spikes to the uninterrupted syllables, whereas its reaction dropped to about 2 spikes for syllables with gaps greater than $4 \mathrm{~ms}$. These different spike responses were very reliable associated with the stimulus type, as can be seen from the small standard deviations. The difference in reaction did not critically depend upon the stimulus intensity. At all intensities tested, the uninterrupted syllable elicited a response with significantly more spikes than the syllable with gaps (Fig. 4). Between 60 and $80 \mathrm{~dB}$ SPL, a syllable with gaps of $5 \mathrm{~ms}$ elicited only 1 spike on the average. (Only at $90 \mathrm{~dB}$ SPL, which normally is not accepted in the behavioural test, the spike count for the syllable with gaps was higher). These results demon-
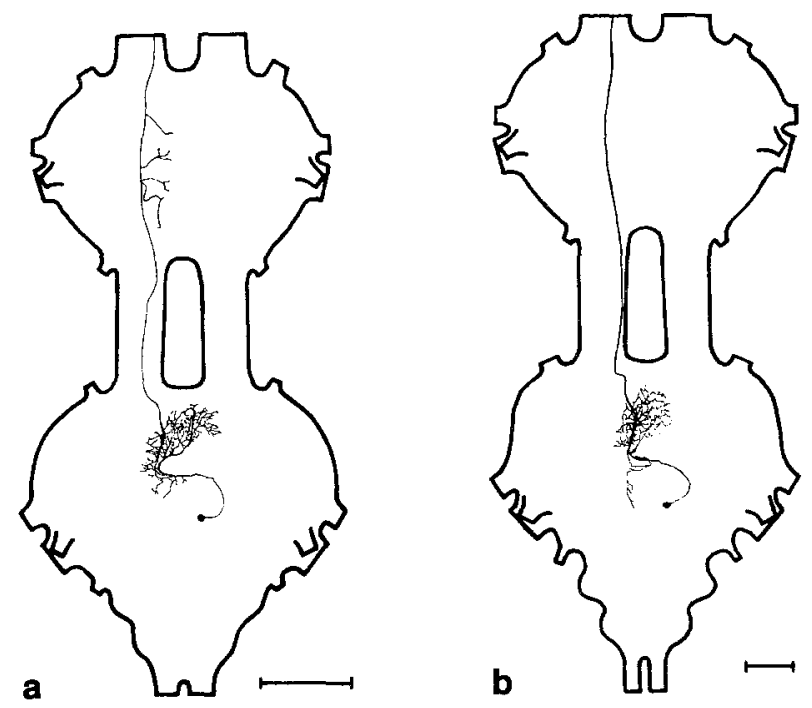

Fig. 2. a Morphology of an ascending auditory interneuron in Ch. biguttulus. Obviously, this neuron is a homologue of the AN4 in Locusta migratoria, which is shown in b. Scale bars: $250 \mu \mathrm{m}$

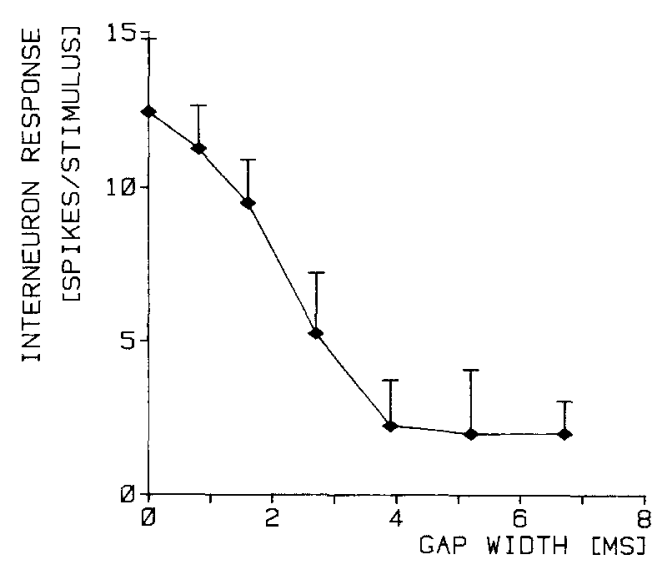

Fig. 3. Reaction of an AN4-neuron in a Ch. biguttulus female to syllables with and without gaps. In the interrupted syllables, the pulse duration was $7.5 \mathrm{~ms}$. Stimulus repetition rate: $1.1 / \mathrm{s}$; $78 \mathrm{~dB}$ SPL. 8 stimulus presentations per point. Bars indicate standard deviations

strate that the sharp decline in reaction in Fig. 3 is not caused by the intensity per time being lower for syllables with gaps. Indeed, as can be seen in recordings from the dendritic branches of the AN4-neuron (see Fig. 5a), the drop in the spike response is due to an inhibition. This neuron type reacts to a stimulus onset first with a pronounced IPSP, followed by an EPSP leading to a spike answer. With the interrupted syllable, however, each pulse onset following a gap induces an IPSP (Fig. 5a, arrows), and the resulting fusion of IPSP's causes a nearly complete suppression of spikes. 


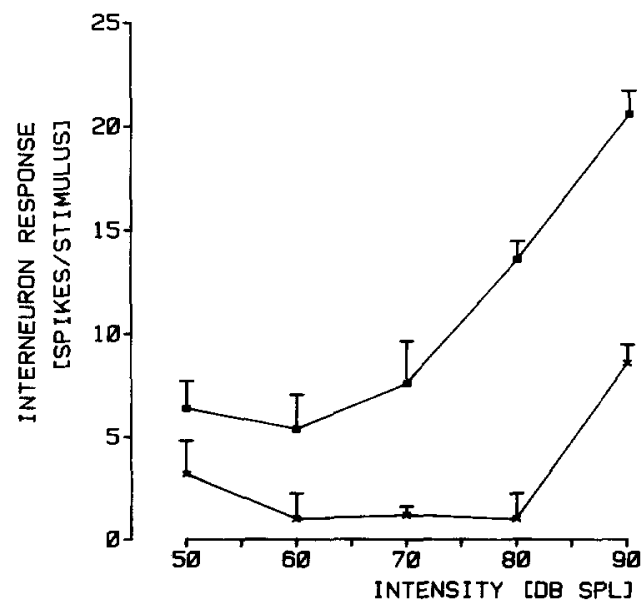

Fig. 4. Reaction of the AN4 to stimuli of different intensities (same cell as in Fig. 3). Two stimuli were tested: an uninterrupted syllable (upper curve) and a syllable with gaps of $5 \mathrm{~ms}$ duration (stimulus repetition rate: $2 / \mathrm{s}, 5$ stimulus presentations per point). In a pair-by-pair comparison, the differences between the respective mean values are highly significant $(P<0.01$ for $50 \mathrm{~dB}$ SPL, $P<0.001$ for all other intensities). Bars indicate standard deviations

Reactions of different specimens of the AN4neuron to stimuli with varied gap widths are shown in Fig. 5 b, c. There was a considerable variation in the maximum spike response (extreme mean values between 6 and 22 spikes/stimulus for the uninterrupted syllable, although most mean values ranged between 10 and 14 spikes/stimulus). However, independent of the absolute spike count, the reaction to syllables with gaps was principally the same in all cases, i.e. a drastic drop in spike response between 2 and 4 ms gap width (Fig. 5b). In this diagram, data are pooled for intensities between 66 and $78 \mathrm{~dB}$ and pulse durations of 5.2 or $7.5 \mathrm{~ms}$. These differences had no systematic effect on the reaction of the neurons. Only at intensities near the threshold (about 50-60 dB) did the reaction change. The overall spike response was reduced (to about 6 spikes for the uninterrupted syllable), and the slope of the curves was, on the average, less steep and shifted toward broader gap widths (Fig. 5 c). Also, a considerably greater variability was observed at low intensities, between individuals as well as between different presentations of the same stimulus. These observations are in agreement with the behavioural results: the drop in reaction shifts to broader gaps at low intensity (O. von Helversen 1979).

In most curves of Fig. 5, a small drop in reaction can be observed even between the uninterrupted syllable and the syllable with $0.8 \mathrm{~ms}$ gaps. This difference is not caused by the gaps, as can be seen from the absence of IPSP's at the pulse onsets in the response to the syllable with $0.8 \mathrm{~ms}$ gaps. Rather, it
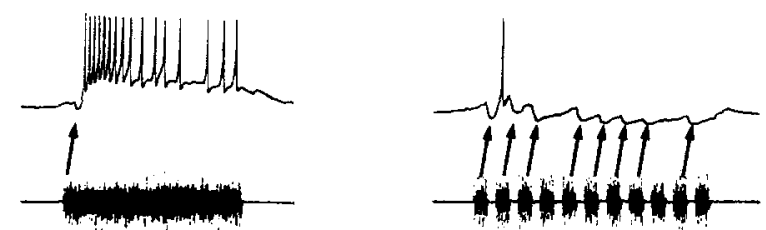

a
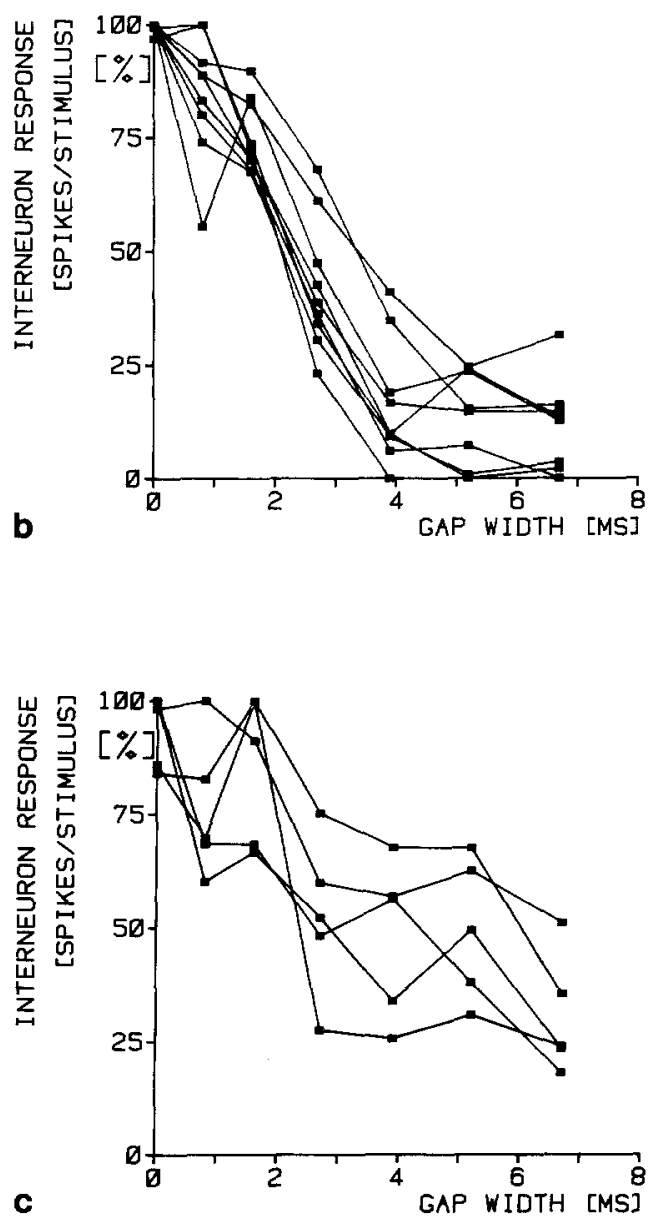

Fig. 5. a Reactions of an AN4 (Ch. biguttulus) to a pulsed and an uninterrupted syllable. Recording from the dendritic region in the metathoracic ganglion. Note IPSP's triggered by the pulse onsets (arrows). Gap width: $5.2 \mathrm{~ms}$. Scale bars: $20 \mathrm{mV}, 50 \mathrm{~ms}$. b and c Reactions of several specimens of the AN4-neuron in 5 Ch. biguttulus females to model songs containing gaps of different width (pulse durations 5 or $7.5 \mathrm{~ms}$ ). Reaction (spikes/ stimulus) is expressed as percentage of the highest reaction (which usually occurred with the uninterrupted syllable). In two preparations the neuron was hyperpolarized by $1.5-3 \mathrm{nA}$ negative current to reduce penetration artefacts. Temperature: $22-25^{\circ} \mathrm{C}$. b Sound pressure between 66 and $78 \mathrm{~dB}$ SPL, i.e. $15-30 \mathrm{~dB}$ above the neuron's threshold. Spike count ranged from 6-22 spikes/uninterrupted syllable (mean values). c Sound pressure between 50 and $60 \mathrm{~dB}$ SPL, i.e. $5-10 \mathrm{~dB}$ above the neuron's threshold. Maximum response between 4 and 11 spikes/syllable 

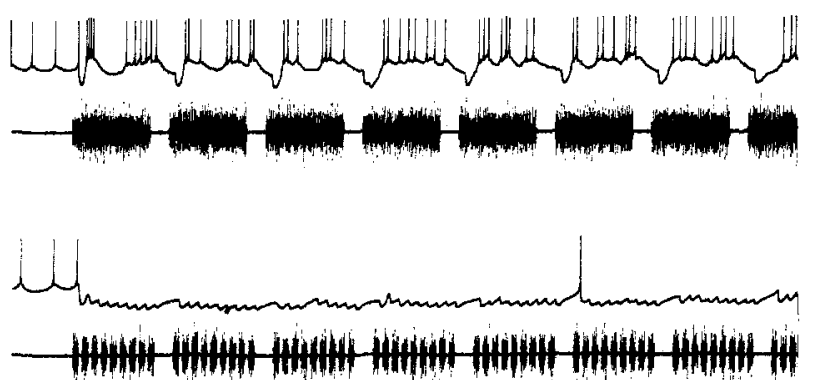

a

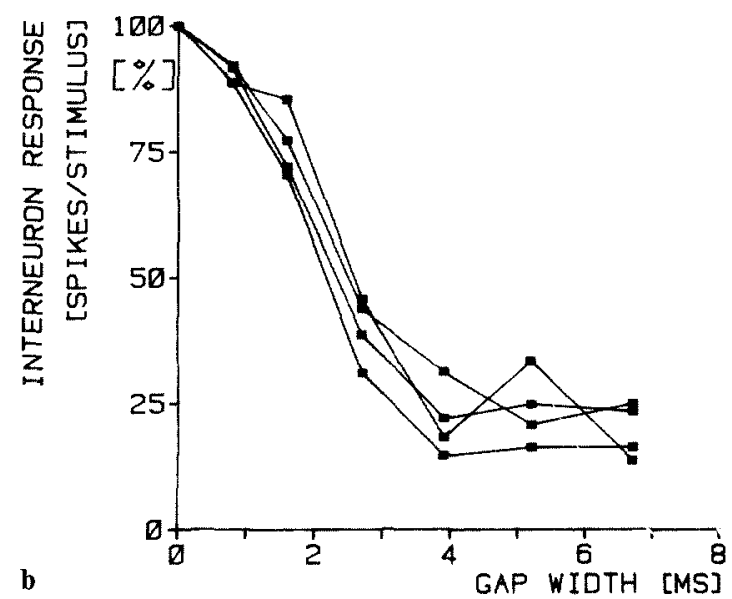

Fig. 6a, b. Reactions of the AN4 of 2 specimens of Locusta migratoria to model songs. a Detail of the reactions to longer models containing uninterrupted syllables or syllables with gaps of $5 \mathrm{~ms}$ duration. Scale bars: $20 \mathrm{mV}, 100 \mathrm{~ms}$. b Reactions to syllables with gaps of different width. Sound pressure between 60 and $66 \mathrm{~dB}$ SPL, i.e. 15-25 dB above threshold. Maximum response $7-16$ spikes/syllable. Data of one individual obtained with $1 \mathrm{nA}$ hyperpolarization. Temperature: $23-25^{\circ} \mathrm{C}$

is due to a slight difference in habituation. The uninterrupted syllable served as a control stimulus and was inserted at every change of the gap width with a longer interval to the preceding stimulus.

A comparison of Figs. 3 and 5 with Fig. 1 reveals - somewhat unexpectedly for a thoracic interneuron - filter characteristics which closely parallel the species-specific behavioural response of Ch. biguttulus females. This striking similarity suggests that the filtering characteristics for temporal parameters of this neuron could be also species-specific. However, this is not the case. As mentioned above, a neuron with similar morphology has been found in Locusta migratoria (AN4, Römer and Marquart 1984). The reactions of the locust's AN4 to these acoustic models are very similar to those of the Ch. biguttulus homologue (compare Figs. 6 and 5).

\section{Discussion}

Participation of the AN4 in the detection of small gaps

The first question, of course, is whether the AN4neuron is actually involved in mediating this very peculiar behavioural response of Ch. biguttulus females. Although a direct proof of this hypothesis is not available at this time (e.g. by elimination experiments (Atkins et al. 1984) or intracellular manipulation in the behaving animal (Schildberger and Hörner 1988)), several arguments speak in favour of a participation of this interneuron in the detection of small gaps by $C h$. biguttulus females.

As mentioned in the Introduction, the information about the presence or absence of gaps has to reach the brain (Bauer and $\mathrm{O}$. von Helversen 1987). There are, in principle, two mechanisms by which an interneuron could transmit this information: The neuron could encode the temporal structure of the stimulus (i.e. the spike distribution presents a copy of the stimulus structure); or, it could perform a weighting of parameter values of the stimulus and react selectively to stimuli fulfilling certain criteria, e.g. not containing gaps.

As concerns the first mechanism, one has to consider the small gap widths detected by the behaving animals (Fig. 1, and $O$. von Helversen 1979) and the spike rates observed in interneurons (at room temperature for tonic reactions up to $250 \mathrm{~Hz}$; higher spike rates, up to $500 \mathrm{~Hz}$, occur only with few neurons in short phasic bursts). For these reasons, it is inconceivable that a single interneuron could encode the stimulus structure in its spike pattern with sufficient precision. Still, the stimulus structure could be represented in the spike pattern of many parallel neurons. Such a mechanism - a synchronization of spikes on parallel fibres - has been proposed for the transfer of information about gaps by the receptor fibres (Ronacher and Römer 1985), since a single receptor fibre is obviously not able to transmit the information about the presence of gaps in the relevant time range. Perhaps, the brain detects the presence of gaps from the reactions of several parallel neurons by a similar mechanism as proposed for the receptor fibres?

According to our data (recordings of approximately 20 types of ascending auditory interneurons) such a mechanism is not likely. There are too few neurons with similar reactions, and, for most of them, a difference in the timing of spikes elicited by an interrupted or an uninterrupted syllable is evident only with very large gaps. Since a song consists of a repetitive pattern, the encoding of the 
stimulus structure could, in principle, be improved by an evaluation of consecutive stimuli (as performed in PST-histograms, in which the responses to many stimulus presentations are summated). However, it is by no means clear whether the CNS of grasshoppers could analyze the timing of spike responses to successive stimuli. Note that the animals already answer to model songs consisting of 10 syllables and, therefore, have to assess a song on the basis of a few syllable repetitions (D. von Helversen 1972).

Hence, neurons with filter properties seem to be required for transporting the information about the presence of small gaps to the brain. There are two further properties which can be expected from a 'filter-neuron'. (i) The reactions of this neuron should be reliable enough that the stimulus type could be inferred by higher centres from few stimulus presentations. (ii) The selectivity of this neuron should be more or less independent of intensity, at least in the behaviourally relevant range. (Some brain neurons in Locusta have been reported to respond selectively to the temporal pattern of the conspecific song (Römer and Seikowsky 1985). However, in these cases, the selectivity was restricted to a very narrow intensity range and thus seems to be very susceptible to minor changes.)

In about 400 recordings from acoustic neurons in the thoracic ganglia of Ch. biguttulus, we found no other ascending neuron as well-suited as the AN4 to transmit the information about small gaps. Indeed, for most ascending interneurons, the spike count for an uninterrupted or an interrupted syllable was more or less the same. In a few neurons, differences were found in spike count elicited by the two kinds of stimuli. However, this effect was always restricted to a small intensity range and, in most cases, was probably an effect of the differing mean intensities of the two types of stimuli. There are, in Ch. biguttulus as well as in L. migratoria (Marquart 1985), some other neurons which show an initial IPSP at stimulus onset (AN3, AN6, 'AN11/12'-types). In these neurons, however, the IPSP is usually not retriggered by each pulse onset, as is the case with the AN4. Only under certain conditions in some examples of these neurons has a summation of IPSPs been observed (in reaction to syllables with gaps), but this effect depended on the side of stimulation and had little or no influence on the spike response.

The strongest arguments for a participation of the AN4 in this task of gap detection are (i) the close agreement in the time range of the behavioural and the neuronal response (Figs. 1 and 5), (ii) the reliability of the difference in response to unin- terrupted and interrupted stimuli, and (iii) the fact that this difference is maintained over the behaviourally relevant intensity range (cf. Fig. 4). Of course, these arguments do not prove that the AN4 in Ch. biguttulus actually participates in the neuronal filter network which determines the characteristics of the IRM. Likewise, we cannot exclude the possibility that other interneurons, too, contribute to the transport of information about the presence of gaps from the thoracic ganglia to the brain. In any case, however, the reactions of the AN4 can be viewed as a model of how the detection of very small gaps could be accomplished in the CNS.

\section{Inferences on the evolution of the IRM}

A remarkable finding is the occurrence of a neuron in the thoracic ganglia whose filter characteristics match the behavioural response of Ch. biguttulus females. In other species, 'temporal filter'-neurons were found only at high levels of the auditory pathway (locusts: Adam 1969; Römer and Seikowski 1985; crickets: Schildberger 1984; frogs: Rose and Capranica 1983, 1984; Walkowiak 1984). Even more surprising is the fact that this neuron has the same response characteristics in L. migratoria, a not closely related species (subfamily Oedipodinae, sensu Dirsh 1975), as in Ch. biguttulus (subfamily Gomphocerinae), compare Figs. 5 and 6. This neuron, therefore, is neither a species-specific acquisition of Ch. biguttulus nor a newly adopted modification of an existing neuron. This observation might shed some light on basic principles of the evolution of such a communication system. Although this neuron in Ch. biguttulus probably constitutes an important step in generating the speciesspecific behaviour, it is itself not species-specific, neither in its morphology nor in its reaction. The filter network which determines the properties of the IRM is species-specific only as a whole, but not necessarily in its elements. This is really not very surprising. Evolution must work upon the basis of existing elements. Our data on other thoracic auditory interneurons provide no evidence of the existence of species-specific neurons at this level (Stumpner and Ronacher, in prep.; see also Hedwig 1986). We hypothesize that the AN4-neuron is common to most acridids and has evolved its physiological characteristics in another, still unknown, context. Thus, it formed a preadaptation for gap detection from which these surprising requirements of Ch. biguttulus females (Fig. 1) could easily evolve. It should be noted that the IRM of male Ch. biguttulus reacts quite differently to 
gaps: Male Ch. biguttulus respond particularly well to model songs with large gaps (and long rise and fall times, see D. and O. von Helversen 1983). The presence of gaps in the song syllables is presumably used by the males to recognize the sex of an answering partner. This leads to the hypothesis that this pre-existing neuron has been integrated into the IRM only by females, or by males and females of Ch. biguttulus with different meaning.

In Locusta several ascending neurons and brain neurons have been investigated with respect to their selectivity to parameters of the conspecific song (Römer and Seikowski 1985). Among them, the AN4 has been tested, too, but without any obvious conclusion as regards its contribution to neuronal filters in this species. To date we do not know what other functions the AN4 could have in other acridid species. These questions should be tackled in a comparative approach.

Acknowledgements. We thank Dagmar and Otto von Helversen for providing the data in Fig. 1 and for helpful comments on the manuscript.

\section{References}

Adam LJ (1969) Neurophysiologie des Hörens und Bioakustik einer Feldheuschrecke (Locusta migratoria). Z Vergl Physiol $63: 227-289$

Atkins G, Ligman S, Burghardt F, Stout JF (1984) Changes in phonotaxis by the female cricket Acheta domesticus L. after killing identified acoustic interneurons. J Comp Physiol A 154:795-804

Bauer M, Helversen O von (1987) Separate localization of sound recognizing and sound producing neural mechanisms in a grasshopper. J Comp Physiol A 161:95-101

Boyan GS (1986) Modulation of auditory responsiveness in the locust. J Comp Physiol A 158:813-825

Dirsh VM (1975) Classification of the acridomorphoid insects. Classey Ltd, Faringdon

Elsner N, Huber F (1969) Die Organisation des Werbegesanges der Heuschrecke Gomphocerippus rufus L. in Abhängigkeit von zentralen und peripheren Bedingungen. Z Vergl Physiol $65: 389-423$

Hedwig B (1986) On the role in stridulation of plurisegmental interneurons of the acridid grasshopper Omocestus viridulus L. II. Anatomy and physiology of ascending and T-shaped interneurons. J Comp Physiol A 158:429-444

Helversen D von (1972) Gesang des Männchens und Lautschema des Weibchens bei der Feldheuschrecke Ch. biguttulus. J Comp Physiol 81:381-422

Helversen D von, Helversen O von (1975) Verhaltensgenetische Untersuchungen am akustischen Kommunikationssystem der Feldheuschrecken. II. Das Lautschema der Artbastarde. J Comp Physiol 104:301-323

Helversen D von, Helversen O von (1983) Species recognition and acoustic localization in acridid grasshoppers: a behav- ioral approach. In: Huber F, Markl H (eds) Neuroethology and behavioral physiology. Springer, Berlin Heidelberg New York, pp 95-107

Helversen O von (1979) Angeborenes Erkennen akustischer Schlüsselreize. Verh Dtsch Zool Ges 1979:42--59

Marquart V (1985) Auditorische Interneurone im thorakalen Nervensystem von Heuschrecken: Morphologie, Physiologie und synaptische Verbindungen. PhD thesis, Bochum

Michelsen A, Larsen ON, Surlykke A (1985) Auditory processing of temporal cues in insect songs: frequency domain or time domain? In: Michelsen A (ed) Time resolution in auditory systems. Springer, Berlin Heidelberg New York, pp $3-$ 27

Pearson KG, Robertson RM (1981) Interneurons coactivating hindleg flexor and extensor motoneurons in the locust. J Comp Physiol 144:391-400

Perdeck AC (1957) The isolating value of specific song patterns in two sibling species of grasshoppers (Ch. brunneus and Ch. biguttulus). Behaviour 12:2-75

Rehbein H, Kalmring K, Römer H (1974) Structure and function of acoustic neurons in the thoracic ventral nerve cord of Locusta migratoria (Acrididae). J Comp Physiol 95:263-280

Römer H (1985) Anatomical representation of frequency and intensity in the auditory system of Orthoptera. In: Kalmring $\mathrm{K}$, Elsner $\mathrm{N}$ (eds) Acoustic and vibrational communication in insects. Parey, Berlin Hamburg, pp 25-32

Römer H, Marquart V (1984) Morphology and physiology of auditory interneurons in the metathoracic ganglion of the locust. J Comp Physiol A 155:249-262

Römer H, Seikowski U (1985) Responses to model songs of auditory neurons in the thoracic ganglia and brain of the locust. J Comp Physiol A 156:845-860

Ronacher B, Römer H (1985) Spike synchronisation of tympanic receptor fibres in a grasshopper (Chorthippus biguttulus L., Acrididae): a possible mechanism for detection of short gaps in model songs. J Comp Physiol A 157:631-642

Ronacher B, Helversen D von, Helversen O von (1986) Routes and stations in the processing of auditory directional information in the CNS of a grasshopper, as revealed by surgical experiments. J Comp Physiol A 158:363-374

Rose G, Capranica RR (1983) Temporal selectivity in the central auditory system of the leopard frog. Science 219: 1087-1089

Rose G, Capranica RR (1984) Processing amplitude modulated sounds by the auditory midbrain of two species of toads: matched temporal filters. J Comp Physiol A 154:211-219

Schildberger K (1984) Temporal selectivity of identified auditory neurons in the cricket's brain. J Comp Physiol A 155:171-185

Schildberger K, Hörner M (1988) The function of auditory neurons in cricket phonotaxis. I. Influences of hyperpolarization of identified neurons on sound localization. J Comp Physiol A (in press)

Stewart W (1978) Functional connections between cells as revealed by dye-coupling with a highly fluorescent Naphthalimide-tracer (Lucifer Yellow). Cell 14:741-759

Surlykke A, Larsen ON, Michelsen A (1988) Temporal coding in the auditory receptor of the moth ear. J Comp Physiol A $162: 367-374$

Walkowiak W (1984) Neuronal correlates of the recognition of pulsed sound signals in the grass frog. J Comp Physiol A 155:57-66 